\title{
Declines in reproductive output in two dolphin populations depleted by the yellowfin tuna purse-seine fishery
}

\author{
Katie L. Cramer ${ }^{1, *}$, Wayne L. Perryman ${ }^{2}$, Tim Gerrodette ${ }^{2}$ \\ ${ }^{1}$ Center for Marine Biodiversity and Conservation, Scripps Institution of Oceanography, University of California, \\ San Diego, La Jolla, California 92093-0208, USA \\ ${ }^{2}$ NOAA Fisheries, Southwest Fisheries Science Center, 3333 North Torrey, Pines Court, La Jolla, California 92037-1023, USA
}

\begin{abstract}
Reproductive data for 2 pelagic dolphin subspecies in the eastern tropical Pacific, the eastern spinner (ES) dolphin Stenella longirostris orientalis and northeastern pantropical spotted (NEPS) dolphin S. attenuata attenuata, were obtained from aerial photographs taken between 1987 and 2003. Two measures of reproductive output were estimated: proportion of adult dolphins with calves ('proportion with calves') and length at which calves disassociated from their mothers ('length at disassociation'). Trends in length at disassociation were investigated to determine if the proportion with calves was affected by possible changes in calving interval, but no changes were found. Proportion with calves for ES dolphins was stable from 1987 to 1993, then declined from 1993 to 2003; proportion with calves for NEPS dolphins decreased steadily from 1987 to 2003. For both species, proportion with calves was related to number of dolphins in the school of the focal species and/or proportion of the school made up of the focal species. For NEPS dolphins, annual number of purse-seine sets on dolphins was a predictor of both proportion with calves and length at disassociation. Because NEPS dolphins are the main species targeted by the fishery, the link between fishing activity and both measures of reproductive output indicates that the fishery has population-level effects beyond reported direct kill. Decline in reproductive output is the proximate cause or one of the proximate causes of the failure of dolphin populations to recover at rates expected after reduction of high bycatch levels.
\end{abstract}

KEY WORDS: Tuna-dolphin issue $\cdot$ Bycatch $\cdot$ Stenella attenuata $\cdot$ Stenella longirostris $\cdot$ Dolphin reproduction $\cdot$ Fishery effects $\cdot$ El Niño effects

\section{INTRODUCTION}

Since the 1950s, purse-seine vessels fishing for yellowfin tuna in the eastern tropical Pacific (ETP) have utilized the association between tuna and certain tropical dolphin species by encircling schools of dolphins in order to capture the tuna swimming beneath them (Perrin 1969). High mortality rates of encircled dolphins, particularly in the early years of the fishery (Wade 1995), led to reductions in the abundance of several dolphin populations (Smith 1983, Wade 1993) and became known as the 'tuna-dolphin issue' (Gerrodette 2002). Two populations of dolphins have been particularly affected by the fishery: the eastern spinner
Stenella longirostris orientalis (ES) dolphin and the northeastern pantropical spotted Stenella attenuata attenuata (NEPS) dolphin. Their abundances have been reduced to an estimated one-third and one-fifth of pre-fishery levels, respectively (Wade et al. 2007). Consequently, these populations have been listed as 'depleted' under the US Marine Mammal Protection Act (US Public Law 92-522). However, by the end of the 1990s, due to a combination of regulations, embargoes, international agreements, scientific studies and efforts by fishermen (Joseph 1994, Gosliner 1999, Hedley 2001), reported dolphin bycatch (dolphins killed by fishing operations) had fallen to $<0.1 \%$ of estimated population sizes (Bayliff 2004). 
In spite of the successful reduction in dolphin bycatch, concerns over the impact of the repeated chase and encirclement of dolphins by this fishery led the US Congress to include additional scientific studies in the 1997 International Dolphin Conservation Program Act (US Public Law 105-42). This law directed NOAA Fisheries to estimate current dolphin abundance and to determine whether tuna fishing operations were having a significant adverse impact on these populations, including effects beyond direct mortality during the chase and encirclement operation. Results reported here are part of that effort. Previous research has shown that 75 to $95 \%$ of lactating females killed in purse-seines do not have their nursing calves with them (Archer et al. 2001, 2004), that repeated chase and encirclement could have negative physiological consequences for individual dolphins (Reilly et al. 2005), and that neither of the 2 dolphin populations considered here is recovering at the expected rate since bycatch was greatly reduced (Gerrodette \& Forcada 2005, Wade et al. 2007).

The failure to recover at the expected rate may be due in part to the cryptic (unobserved) mortality of calves that occurs when nursing calves are separated from their mothers during fishing operations (Archer et al. 2001, Edwards 2006, Noren \& Edwards 2007). The swimming ability and stamina of young calves are inferior to those of adults, and the number of sets on dolphins has been increasing over the past decade (Bayliff 2004). Besides cryptic effects of the fishery, ecosystem changes, interspecific population dynamics effects, and underreporting of dolphin bycatch are considered further hypotheses for the lack of recovery (Gerrodette \& Forcada 2005, Wade et al. 2007).

Using medium-format aerial photography, we collected and analyzed reproductive data for these depleted populations between 1987 and 2003. Two measures of reproductive output were estimated: proportion of adult dolphins with calves ('proportion with calves') and the body length at which calves disassociated from their mothers ('length at disassociation'). As trends in proportion with calves' values are indicative of changes in calving interval and/or calf survival rate, assessing trends in length at disassociation allowed us to determine whether changes in proportion with calves could be attributed to changes in calving interval.

Previous studies have assessed reproduction based on dolphins killed by US-based purse-seine vessels in the 1970s and 1980s (Perrin et al. 1976, 1977, Henderson et al. 1980, Perrin \& Henderson 1984, Myrick et al. 1986, Chivers \& Myrick 1993). Direct information on current reproduction is lacking, because vessels from the USA no longer set on dolphins, and observers on vessels which do set on dolphins, primarily from
Mexico, Ecuador, and Venezuela, do not collect biological samples from the dolphins killed. The present study is the first to assess trends in reproduction for these populations independently of fishery-derived data, which are known to contain sampling biases (Powers \& Barlow 1979, Barlow \& Hohn 1984).

\section{MATERIALS AND METHODS}

Aerial photography. Dolphin counts and measurements were derived from medium-format $(11.4 \times$ $11.4 \mathrm{~cm}$ ) vertical aerial photographs of ES (Stenella longirostris orientalis) and NEPS (S. attenuata attenuata) dolphins collected between 1987 and 2003 during the months of August to December. Photographs were taken with 2 military reconnaissance cameras mounted below the hull of a Hughes 500D helicopter. The helicopter was carried aboard the NOAA research vessel 'David Starr Jordan' as part of the population surveys described above. Photographic sampling was conducted when sun angle and sea conditions permitted. Sampling occurred throughout the study area in all years except for 2: sampling was concentrated in the southern part of the species' range in 1992 and in the northern part of the range in 1993. One camera was typically outfitted with a wide-angle lens and color film for the purposes of counting as many animals as possible in a school and of identifying them to species and by swimming association type ('Count data' below). The other camera was typically outfitted with a telephoto lens and high-resolution black and white film to produce length measurements from individual animals in a school ('Measurement data' below).

A series of photographic passes was made over the dolphin school during which the cameras took overlapping photographs at an approximate rate of 1.7 frames $\mathrm{s}^{-1}$, resulting in 4 to 6 frames of each animal per pass. Each pass was reviewed, and the pass that contained the largest number of dolphins swimming parallel to and near the sea surface was selected for analysis. Individual frames taken from the highest quality pass were analyzed under a magnifying scope mounted above a light table in the laboratory. Approximately $80 \%$ of the photographic passes analyzed contained $>90 \%$ of the dolphins in a school. Further details of photographic methods are described in Perryman \& Lynn (1993, 1994).

Count data. All clearly visible individual dolphins in the selected photo pass were counted and scored as engaged in either a mother-calf swimming formation or a non-mother-calf swimming formation. The mother-calf swimming formation (Fig. 1) was identified by 2 criteria: (1) the presence of 2 animals of unequal length, with the smaller dolphin (the 'calf') 


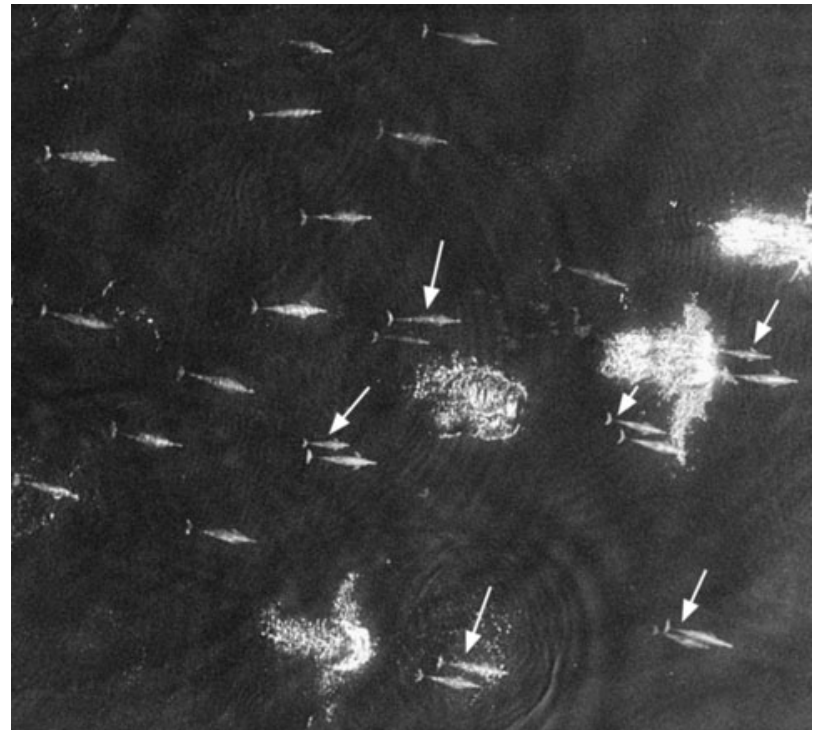

Fig. 1. Stenella longirostris orientalis. Aerial photograph of portion of eastern spinner dolphin school. Mother-calf pairs are indicated by arrows

generally $\leq 0.75$ the length of the larger dolphin (the 'mother') and (2) the closer proximity of the pair to one another than to any other surrounding dolphins. Calves were observed in 1 of 4 swimming positions: (1) oriented on the same horizontal plane as the mother, with the shortest distance between animals located near the dorsal fin region of the mother ('echelon position'); (2) oriented below the mother, with melon near the abdomen of the mother ('infant position'; Gubbins et al. 1999); (3) trailing behind the mother; or (4) not associated closely with another noncalf dolphin. Calves in the last position were very rarely encountered.

All other types of swimming associations involving adult-sized animals were classified as non-mother-calf swimming formations. These were generally much looser associations between individuals and included various types of spatial arrangements between nearest neighbors, although echelon-type swimming among adults was also common. Dolphin pairs which were not clearly visible in the images and for which we could not determine with certainty the presence or absence of a calf were excluded from the counts. Lone animals which were not engaged in a distinct swimming association with another dolphin were assigned to adult or calf categories based on their size relative to other animals in the photograph, as discussed above.

For a subset of mother-calf and non-mother-calf pairs, we measured nearest-neighbor distances to quantify the difference between the 2 swimming associations. Individuals who appeared to be engaged in mating behavior or who were not part of a cohesive subgroup of the school were not included in these measurements.

Proportion with calves. For each school, the proportion of dolphins associated with a calf was computed as the ratio of calves/non-calves. As factors that were easily measurable and would most likely have the greatest impact on the observed proportion with calves for each school, we considered: (1) year of the photograph, (2) group size (total school size if singlespecies school or number of animals of focal species if mixed-species school), (3) species proportion (proportion of individuals of focal species in the school), (4) distance from shore, (5) multivariate El Niño-Southern Oscillation (ENSO) index averaged over all monthly values for that year ('MEI'; Wolter \& Timlin 1993, 1998), and (6) number of purse-seine sets on all dolphin species for that year ('dolphin sets'; Bayliff 2004). Distance from shore/MEI and dolphin sets were included as indicators of effects of habitat quality and fishing activity on dolphin reproduction, respectively. Group size and species proportion were included in the analyses so that the effects of school composition versus habitat quality and fishing activity on dolphin reproduction could be assessed. Including the additional 5 factors beyond the year of the photograph also allowed us to separate out their individual effects on dolphin reproduction and the extent to which each contributed to any observed temporal trend in reproduction.

Group size and species proportion were usually estimated by shipboard observers, but data from the aerial photographs were used if the entire school had been photographed. In a few cases, estimates by observers in the helicopter were used when neither of the other 2 types of data was available. Shipboard observer estimates were corrected for individual-observer bias (Gerrodette et al. 2002).

Generalized additive models of the form $y=a+\Sigma_{i}$ $S_{i}\left(X_{i}\right)$ were used to estimate the effects of predictor variables $x$ on the outcome variable $y$, the proportion with calves for each dolphin school $i$. The functions $s$ are smooth functions of the predictor variables $x$, the summation is over the number of predictors, and $a$ is a constant. Additive models (Hastie \& Tibshirani 1990) provide generality and flexibility in modeling (possibly) nonlinear effects; models were fit with the gam function in S-Plus. For each analysis the default smoothing spline method, a Gaussian error distribution, an identity link, and a weight (the number of sampled non-calf dolphins in the school) were specified. Because a few very large schools dominated the model fitting, we restricted the analysis to schools of 600 dolphins or fewer. This eliminated 6 of 88 ES and 3 of 124 NEPS dolphin schools. 
Model selection was based on Akaike's information criterion (AIC) (Akaike 1973, Burnham \& Anderson 1998). We manually explored models of single and multiple predictors with up to 3 degrees of freedom (nonlinearity). For both species, several different models, each with a single predictor, had similar lowest AIC values. These single-predictor models did not, however, lead to consistent predictor effects across models, so model averaging of these single-predictor models was not found to be a useful approach (Schnute \& Hilborn 1993). Instead, we focused on a set of 5 models with several predictors whose effects were non-zero and consistent regardless of which other predictors were included. We considered this set of models biologically plausible and used AIC to rank and weight the relative influence of each model to produce model-averaged results. Uncertainty was estimated by resampling schools with replacement. From 1000 bootstrap samples, 85\% confidence intervals were estimated by the 75 th lowest and highest values of each effect.

Measurement data. Dolphins swimming parallel to and close enough to the sea surface so that both the tip of the rostrum and straight edge of the flukes were visible were selected for measurement. Original black and white film negatives were converted to digital format, and body length for each dolphin was measured as the distance from the tip of the rostrum to the trailing edge of the tail flukes using Image Pro Plus 4.0 software (Media Cybernetics, 1998). We followed the procedures outlined in Perryman \& Westlake (1998) to convert image lengths to real lengths and to correct for possible biases in measurements due to lateral tilting of the helicopter. Biases in measurement originating from errors in altitude measurement were avoided by calibrating the radar altimeter readings at least once every $3 \mathrm{wk}$ throughout the photographic sampling period (Gilpatrick 1996). Accuracy tests conducted on dolphin-sized targets resulted in a standard error of approximately $2 \mathrm{~cm}$ (Perryman \& Lynn 1993).

Length at disassociation. For each animal measured, we also recorded whether it was a calf swimming with its mother (the smaller animal in a mother-calf association) or not. Generalized additive models, as described above, were used to estimate the probability that a calf would be associated with an adult, as a function of length, year, MEI, and dolphin sets. MEI and dolphin sets were included as indicators of effects of habitat quality and fishing activity on length at disassociation, respectively, and year was included to determine if temporal changes in length at disassociation could be partially responsible for any observed changes in proportion with calves. Since the outcome variable was binomial (each calf associated with an adult or not), we used logistic regression (logit link function and binomial error distribution). Length at disassociation was defined as the length at which the probability of being in mother-calf formation was 0.5. Model-averaged results and uncertainty were estimated using the procedure described for analyses of proportion with calves. Lengths were converted to ages using updated, unpublished versions of published growth curves for both ES (original: Perrin \& Henderson 1984; updated: J. Larese unpubl. data) and NEPS (original: Bright \& Chivers 1991; updated: S. Chivers unpubl. data) dolphins. Length to age conversions were computed assuming there had been no change in calf growth rates between the times when data were collected from fishery kills for growth curves (1972 to 1993) and for our study (1987 to 2003). Given that population sizes for both species were relatively constant during this period (Hoyle \& Maunder 2004, Gerrodette \& Forcada 2005), no density-dependent changes in calf growth rates would be expected.

Geographic bias. Tests for spatial clustering of: (1) location of schools counted or measured, (2) proportion with calves, and (3) mean calf length were conducted to determine if the opportunistic (non-random) manner of photographic sampling of schools resulted in a spatial bias in the proportion with calves and length at disassociation results. Comparison of expected and observed nearest-neighbor distances were used to determine the degree to which schools were spatially distributed in a non-random manner. Moran's I spatial autocorrelation statistic (Moran 1950) was computed to determine if the proportion with calves and mean calf length by school were spatially distributed in a non-random manner. These tests were performed using ArcGIS 9.0 (ESRI, 2004).

\section{RESULTS}

\section{Sample size and distribution}

Dolphins were counted from photographs of 160 schools located throughout the geographic range of both populations (Fig. 2). A total of 11466 ES dolphins from 88 schools and 9700 NEPS dolphins from 124 schools were counted (Table 1). The sum of the total number of schools of each species exceeds the total number of schools (160) because some schools contained both species. A total of 1280 ES dolphins from 33 schools and 298 NEPS dolphins from 31 schools were measured (Table 1). Due to a combination of photographic quality, environmental conditions, and dolphin swimming behavior, sometimes only one of the species in a mixed-species school could be counted and/or measured. 


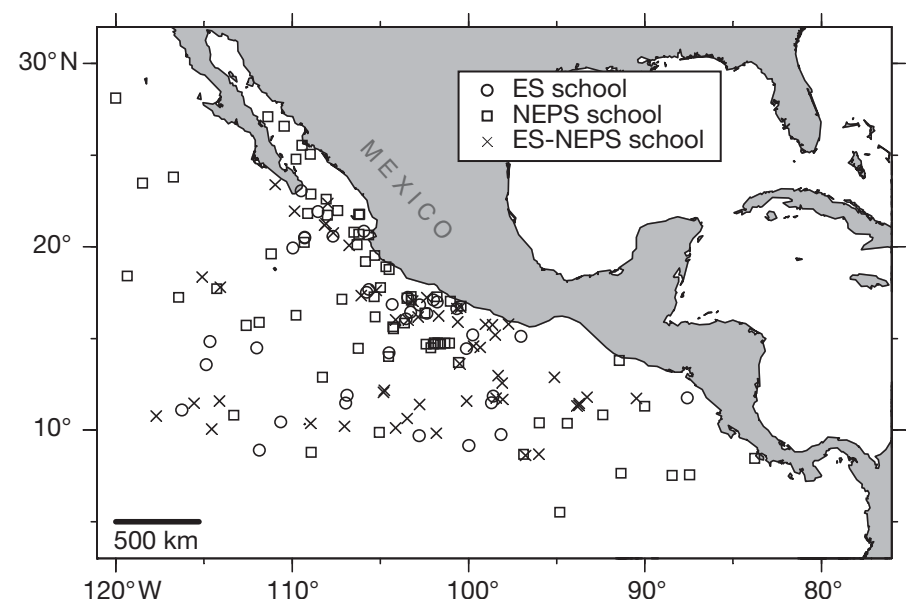

Fig. 2. Stenella longirostris orientalis and $S$. attenuata attenuata. Locations of all eastern spinner (ES) and northeastern pantropical spotted (NEPS) dolphin schools analyzed in the present study

\section{Mother-calf swimming association}

For both dolphin populations, the mean nearestneighbor distance was much smaller and less variable for mother-calf pairs than for non-mother-calf pairs (Fig. 3). The mean distance for mother-calf pairs was $14.3 \mathrm{~cm}(\mathrm{SE}=1.7 \mathrm{~cm}, \mathrm{n}=30)$ for $\mathrm{ES}$ and $36.6 \mathrm{~cm}(\mathrm{SE}=$ $4.0 \mathrm{~cm}, \mathrm{n}=30$ ) for NEPS dolphins. The mean distance between non-mother-calf nearest neighbors was $171.5 \mathrm{~cm}(\mathrm{SE}=19.5 \mathrm{~cm}, \mathrm{n}=30)$ for $\mathrm{ES}$ and $109.3 \mathrm{~cm}$ $(\mathrm{SE}=18.7 \mathrm{~cm}, \mathrm{n}=30$ ) for NEPS dolphins.

\section{Proportion with calves}

For ES dolphins, when considered as individual factors, year, school size, and distance from shore may
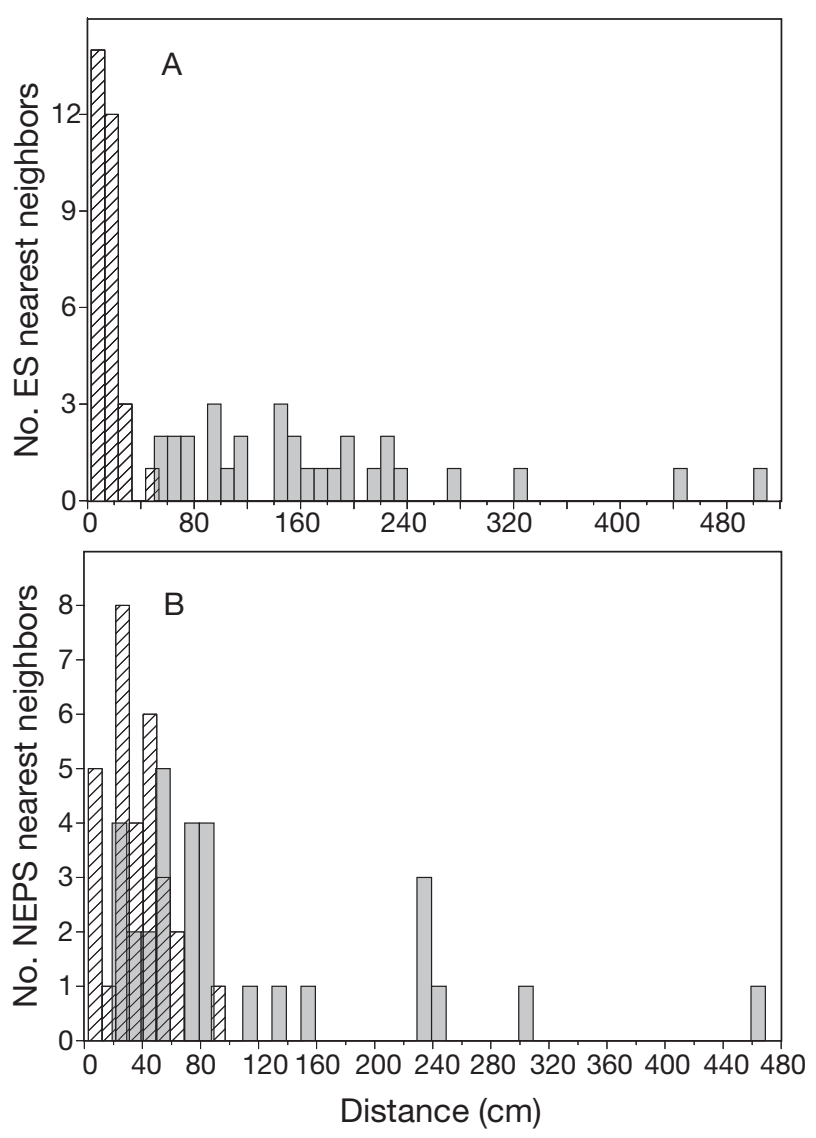

Fig. 3. Stenella longirostris orientalis and $S$. attenuata attenuata. Distributions of nearest horizontal distances within mother-calf pairs (hatched) and between individuals not in mother-calf pairs (solid) of: (A) eastern spinner (ES) and

(B) northeastern pantropical spotted (NEPS) dolphins

have some association with the proportion with calves. The most parsimonious additive models included year, school size, and species proportion as important predictors of the proportion with calves (Table 2A). The

Table 1. Stenella longirostris orientalis and S. attenuata attenuata. Summary of eastern spinner (ES) and northeastern pantropical spotted (NEPS) dolphins counted and measured from aerial photographs

\begin{tabular}{|c|c|c|c|c|c|c|c|c|}
\hline \multirow[t]{3}{*}{ Year } & \multicolumn{4}{|c|}{ ES dolphin } & \multicolumn{4}{|c|}{ NEPS dolphin } \\
\hline & \multicolumn{2}{|c|}{ Counted } & \multicolumn{2}{|c|}{ Measured } & \multicolumn{2}{|c|}{ Counted } & \multicolumn{2}{|c|}{ Measured } \\
\hline & Schools & Animals & Schools & Animals & Schools & Animals & Schools & Animals \\
\hline 1987 & 8 & 489 & 1 & 34 & 18 & 1162 & - & - \\
\hline 1988 & 9 & 1568 & 7 & 632 & 7 & 700 & 3 & 45 \\
\hline 1989 & 11 & 1254 & 4 & 186 & 10 & 851 & 2 & 60 \\
\hline 1990 & 11 & 943 & 7 & 221 & 7 & 582 & 3 & 13 \\
\hline 1992 & 8 & 2073 & 2 & 42 & 14 & 1134 & 8 & 80 \\
\hline 1993 & 8 & 684 & 1 & 12 & 15 & 1718 & 8 & 63 \\
\hline 1998 & 14 & 2320 & - & - & 17 & 971 & - & - \\
\hline 1999 & 2 & 101 & - & - & 8 & 272 & - & - \\
\hline 2000 & 8 & 1458 & 3 & 74 & 15 & 1459 & 3 & 19 \\
\hline 2003 & 9 & 576 & 8 & 79 & 13 & 851 & 4 & 18 \\
\hline Totals & 88 & 11466 & 33 & 1280 & 124 & 9700 & 31 & 298 \\
\hline
\end{tabular}


model-averaged estimated partial effects were a nonlinear decreasing effect for year, a linear increasing effect for school size, and a linear decreasing effect for species proportion (Fig. 4). Numerically, the estimated proportion of adult dolphins with calves: (1) was approximately constant from 1987 to 1993 at about 0.145 , then declined to 0.111 by 2003 ; (2) increased from 0.124 to 0.174 as school size increased from 8 to 541 dolphins; and (3) decreased from 0.166 to 0.116 as the proportion of the school composed of ES dolphins increased from 4 to $100 \%$. The overall mean proportion of ES dolphins with calves was 0.135 - that is, on average, about $14 \%$ of ES dolphins had a calf swimming with them.

For NEPS dolphins, when considered as individual factors, year, school size, species proportion, and dolphin sets may have some association with the proportion with calves. The most parsimonious additive models included year, school size, MEI, and dolphin sets as important predictors of the proportion of dolphins with calves (Table 2B). The estimated partial effects for year, school size, and dolphin sets were linear decreasing functions, while the effect for MEI was convex (Fig. 5). Numerically, the estimated proportion with calves: (1) decreased from 0.169 to 0.133 from 1987 to 2003, (2) decreased from 0.164 for a school size of 15 , to 0.118 for a school size of 456, and (3) decreased from 0.182 to 0.131 when the number of dolphin sets increased from 7000 to 13800 . The proportion of dolphins with calves was 0.156 for low MEI

Table 2. Stenella longirostris orientalis and S. attenuata attenuata. Model structure for additive models of the proportion with calves for: eastern spinner (ES) and northeastern pantropical spotted (NEPS) dolphins. Term in model: data are dfs for each term-1: linear model, 2: an additional nonlinear df. For each species, the selected model is listed first. AIC: Akaike's information criterion; MEI: multivariate ENSO index

\begin{tabular}{|c|c|c|c|c|c|c|}
\hline \multicolumn{6}{|c|}{ - Term in model } & \multirow[t]{2}{*}{$\mathrm{AIC}$} \\
\hline Year & Group size & Proportion & Distance & MEI & Sets & \\
\hline \multicolumn{7}{|c|}{ ES dolphin } \\
\hline 2 & 1 & 1 & & & & 30.68 \\
\hline 2 & 1 & 1 & 1 & & & 32.41 \\
\hline 2 & 1 & 1 & & 1 & & 32.47 \\
\hline 2 & 1 & 1 & & & 1 & 32.64 \\
\hline 2 & 1 & 1 & 1 & 1 & & 34.21 \\
\hline 2 & 1 & 1 & 1 & & 1 & 34.37 \\
\hline 2 & 1 & 1 & & 1 & 1 & 34.38 \\
\hline 2 & 1 & 1 & 1 & 1 & 1 & 36.13 \\
\hline \multicolumn{7}{|c|}{ NEPS dolphin } \\
\hline 1 & 1 & & & 2 & 1 & 48.97 \\
\hline 1 & 1 & & & 1 & 1 & 49.16 \\
\hline 1 & 1 & 1 & & 2 & 1 & 50.79 \\
\hline 1 & 1 & & 1 & 2 & 1 & 50.95 \\
\hline 1 & 1 & 1 & 1 & 2 & 1 & 52.76 \\
\hline
\end{tabular}

values (La Niña conditions), and increased to approximately 0.168 for intermediate MEI values (normal conditions). At the highest MEI values (El Niño conditions), the proportion with calves decreased to 0.127 . The overall mean proportion with calves was 0.153 that is, on average, $15 \%$ of adult NEPS dolphins had a calf swimming with them.

\section{Length at disassociation}

For ES dolphins, body length was the most important predictor of the probability of an individual being associated with an adult. The model-averaging results included a nearly linear effect for length and very small numerical effects for year, MEI, and number of dolphin sets (Fig. 6). The weak effects for these 3 predictor variables are due to their inclusion in only 1 or 2 of the 5 most parsimonious models (Table 3A). Length at dissociation was estimated as $141.8 \mathrm{~cm}$, or about $2 \mathrm{yr}$ of age.

For NEPS dolphins, length was the most important predictor of the probability of an individual being associated with an adult, modeled as a linear effect (Fig. 7). Number of dolphin sets had a smaller, but important effect, and MEI had a very weak effect. Length at disassociation decreased with number of dolphin sets, from $167 \mathrm{~cm}(\sim 6 \mathrm{yr})$ with about 7000 sets in 1993 to $140 \mathrm{~cm}(\sim 3 \mathrm{yr})$ with nearly 14000 sets in 2003 (Fig. 8).

\section{Geographic bias}

As anticipated, schools were spatially clustered for ES dolphins (observed/expected nearest-neighbor distance $=0.68, \mathrm{p}<0.01$ ) and NEPS dolphins (observed/ expected nearest-neighbor distance $=0.64, \mathrm{p}<0.01$ ) However, values of the proportion with calves were not spatially clustered for ES dolphins (Moran's I: expected $=-0.01$, observed $=-0.03, p>0.1$ ) nor for NEPS dolphins (expected $=-0.01$, observed $=-0.01$, $\mathrm{p}>0.1$ ). Similarly, average calf length was not spatially clustered for ES dolphins (expected $=-0.01$, observed $=0.03, \mathrm{p}>0.1$ ) nor for NEPS dolphins (expected $=$ -0.01 , observed $=0.00, \mathrm{p}>0.1$ ).

\section{DISCUSSION}

\section{Proportion with calves}

The proportion with calves in a population is a potentially complicated function of all vital rates (Goodman 1984). Given the relatively constant population sizes during the period of study, the proportion 

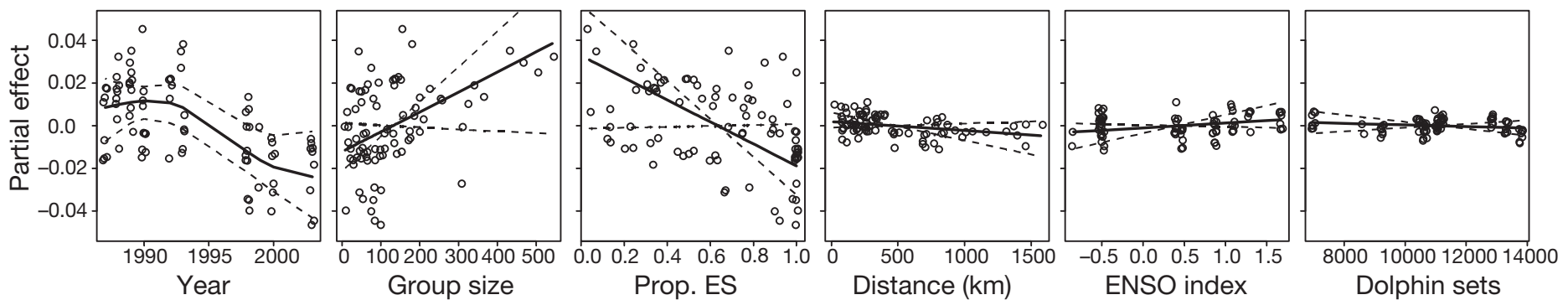

Fig. 4. Stenella longirostris orientalis. Model-averaged partial effects of year, number and proportion of eastern spinner (ES) dolphins in the school, distance offshore, multivariate ENSO index, and annual number of dolphin sets. Solid black lines: estimated partial additive effects, centered on the mean value. Dashed lines: $85 \%$ CIs on the partial effects; symbols are fitted values centered on the mean (differences between symbols and solid line are residuals). A few large outliers are not shown
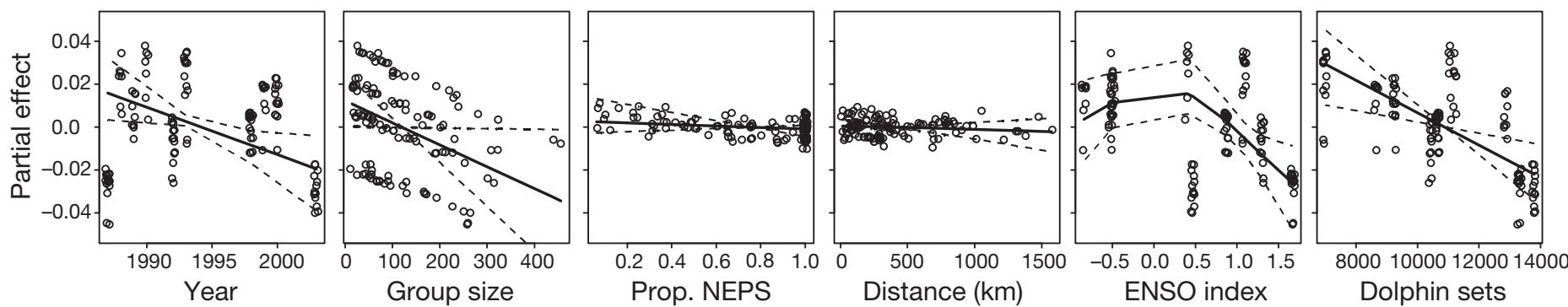

Fig. 5. Stenella attenuata attenuata. Model-averaged partial effects of year, number and proportion of north-eastern pantropical spotted (NEPS) dolphins in the school, distance offshore, multivariate ENSO index, and the annual number of dolphin sets. Solid black lines: estimated partial additive effects, centered on the mean value. Dashed lines: $85 \%$ confidence intervals on the partial effects. Symbols are fitted values centered on the mean (differences between symbols and solid line are residuals), with a small amount of random noise added on the horizontal scale to show individual points more clearly. A few large outliers are not shown
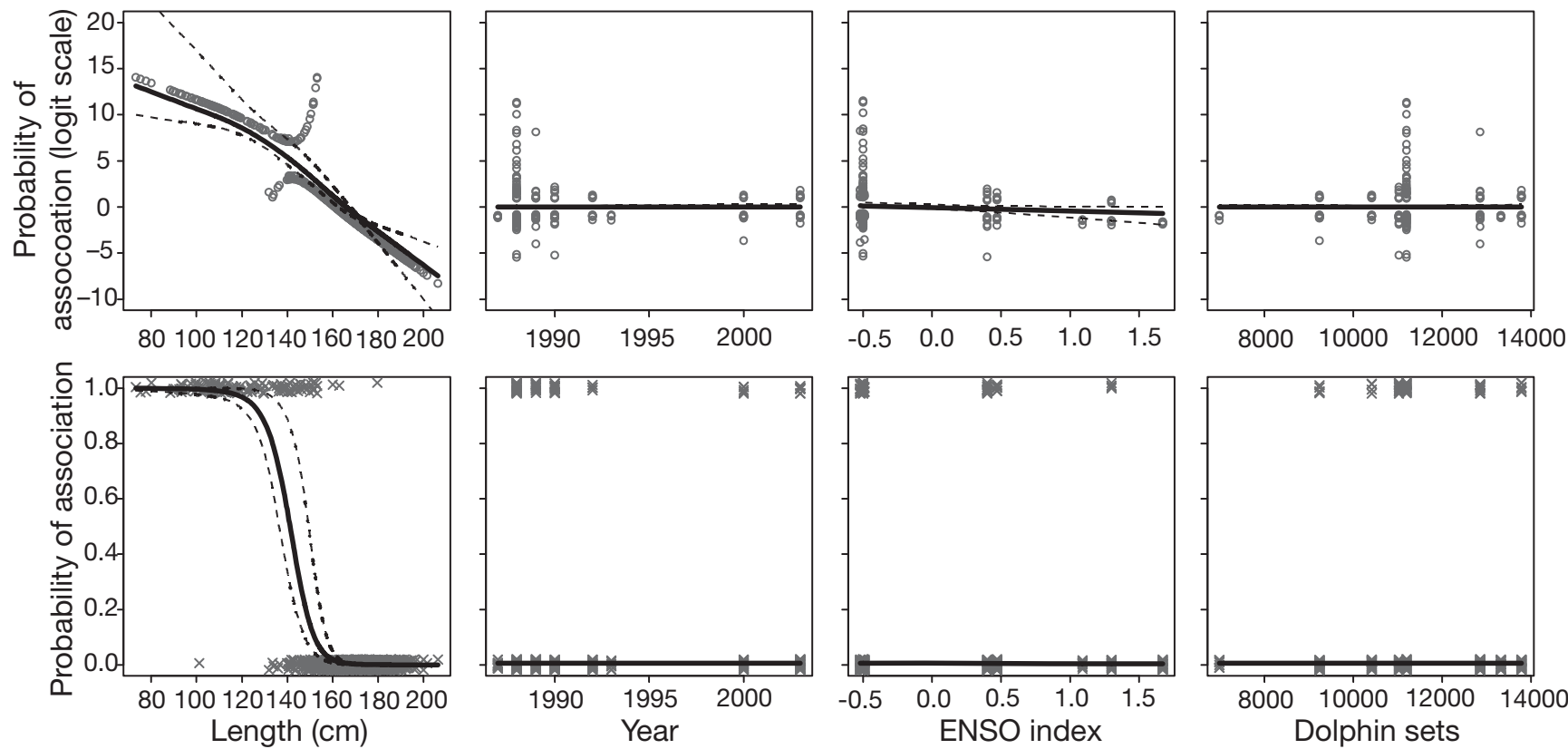

Fig. 6. Stenella longirostris orientalis. Model-averaged results of the probability of association with an adult as a function of length, year, multivariate ENSO index, and annual number of dolphin sets. Solid lines: model estimates; dashed lines: $85 \%$ CIs. Top row: partial effects on the logit scale; symbols: fitted values centered on the mean (differences between symbols and solid line are residuals). Bottom row: partial effects on the scale of the data; symbols show the data with a small amount of random noise added to show individual points more clearly. A few large outliers are not shown 
Table 3. Stenella longirostris orientalis and S. attenuata attenuata. Model structure for additive models of the length at calf disassociation from mother for: eastern spinner (ES) and northeastern pantropical spotted (NEPS) dolphins. Term in model: data are dfs for each term -1: linear model, 2: additional nonlinear df. For each species, the selected model is listed first. AIC: Akaike's information criterion; MEI: multivariate ENSO index; 1 : linear model; 2 : additional linear $\mathrm{df}_{i}$ 3: two additional nonlinear dfs

\begin{tabular}{|lcccc|}
\hline \multicolumn{4}{c}{ Term in model } & \multirow{2}{*}{ AIC } \\
\cline { 1 - 3 } Length & Year & MEI & Dolphin sets & \\
\hline ES dolphin & & & & \\
3 & & 1 & & 209.56 \\
2 & & 1 & & 209.60 \\
2 & & & 1 & 210.44 \\
2 & 1 & & & 212.43 \\
2 & & 1 & \\
NEPS dolphin & & 2 & 1 & 101.97 \\
1 & & & & 103.28 \\
1 & 1 & & & 111.21 \\
1 & & 2 & & \\
1 & & & & \\
1 & & & & \\
\hline
\end{tabular}

with calves can be interpreted as a general index of reproductive output. A decrease in proportion with calves could be caused by a decrease in birth rate, a decrease in calf survival, or both, but is not likely to result from a change in calf growth rate (as discussed in 'Length at disassociation, Materials and methods').

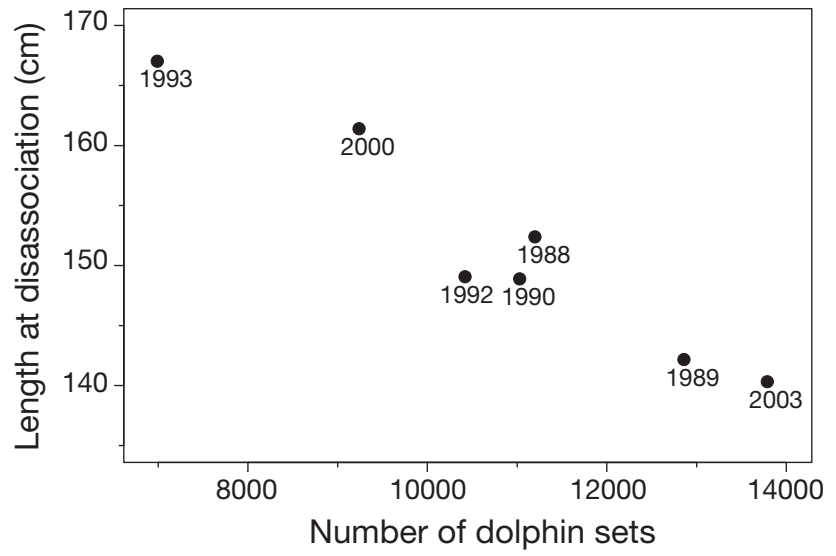

Fig. 8. Stenella attenuata attenuata. Model-averaged estimates of length at disassociation as a function of number of dolphin sets for each year that dolphin calves were measured

The relationship between species proportion and group size and proportion with calves differed between the 2 species. Both commonly form mixedspecies schools in the ETP, but not with equal frequency. While $79 \%$ of ES dolphins sighted occurred in mixed-species schools, only $56 \%$ of NEPS dolphins did. For ES dolphins, proportion with calves increased with group size and proportion of the school composed of NEPS dolphins (Fig. 4). For NEPS dolphins, proportion with calves decreased with group size and was not affected by species composition of the school (Fig. 5).
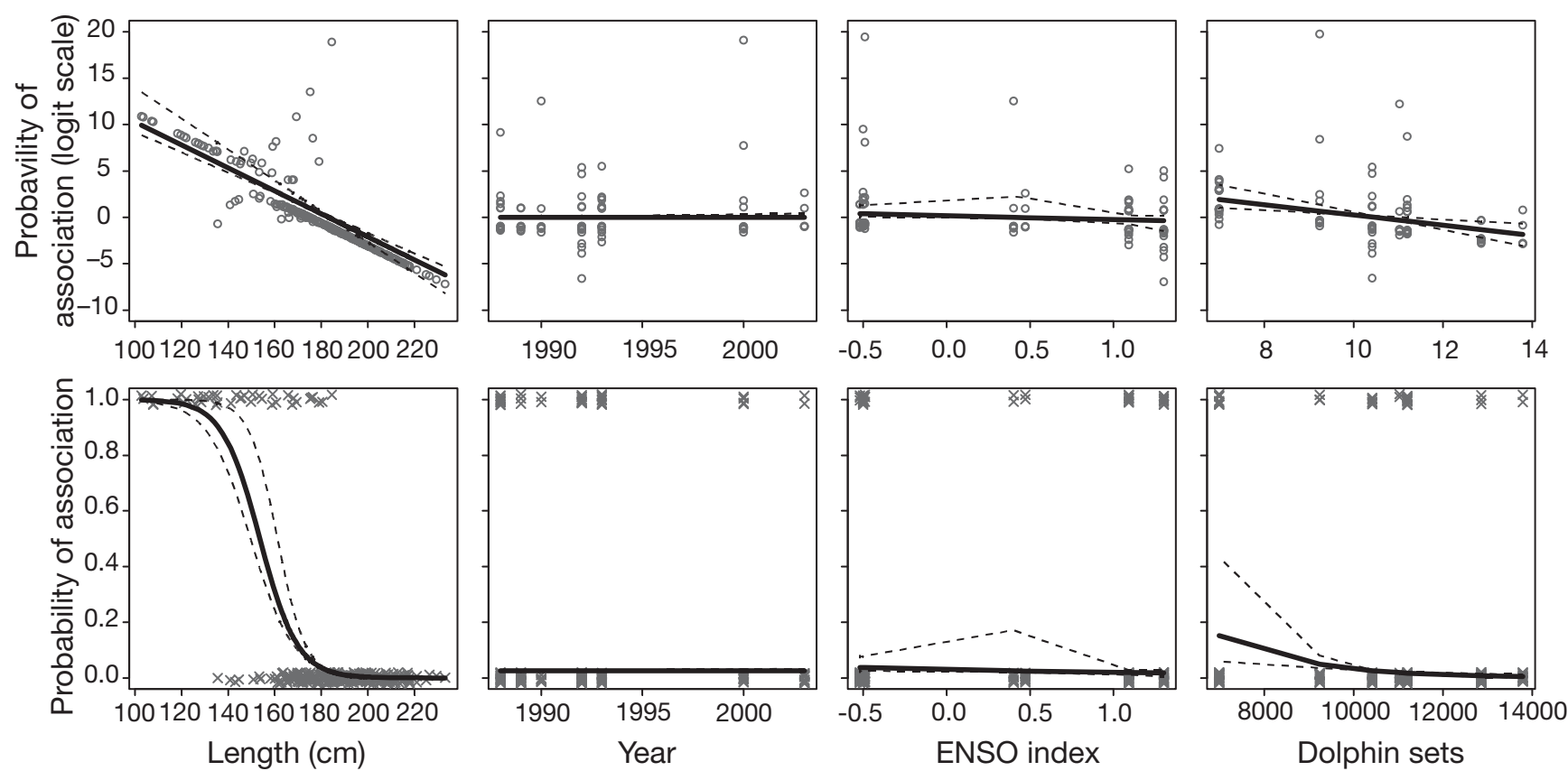

Fig. 7. Stenella attenuata attenuata. Model-averaged results of the probability of association with an adult as a function of length, year, multivariate ENSO index, and annual number of dolphin sets. Solid lines: model estimates; dashed lines: 85 \% CIs. Top row: partial effects on the logit scale; symbols: fitted values centered on the mean (differences between symbols and solid line are residuals). Bottom row: partial effects on the scale of the data; symbols show the data with a small amount of random noise added to show individual points more clearly. A few large outliers are not shown 
As species proportion had a greater effect on proportion with calves for ES dolphins than for NEPS dolphins, mothers and calves from the former may benefit more from forming interspecific associations than mothers and calves from the latter. In the majority of mixed-species schools, NEPS dolphins were located primarily in the front and periphery of the school (K. L. Cramer pers. obs.), suggesting ES dolphins may receive some form of protection by associating with the larger-bodied NEPS dolphins. Similarly, ES mothers and calves may benefit more from overall larger school sizes (possibly enhanced by the presence of NEPS dolphins) than NEPS mothers and calves.

The schools sampled in the photographs are ephemeral groupings of individuals, variable in space and time due to the fission-fusion type herding behavior typical of pelagic and semi-pelagic dolphin species (Norris \& Dohl 1980, Norris \& Johnson 1994, Scott 1998, Chivers \& Scott 2002, Ward 2005). Therefore, although school size and species proportion are statistical predictors of proportion with calves, we do not interpret them as determinates of reproductive output. Rather, mothers with calves tend to be associated with groups of certain sizes and species proportions. The mean school size for these populations observed during abundance surveys was about 100 dolphins, but can range from a few animals to several thousand (Gerrodette \& Forcada 2005). Therefore, relevant demographic units are probably groups that are much smaller than observed schools (Norris \& Johnson 1994).

While proportion with calves was related to MEI -values for the NEPS dolphin, it was not for the ES dolphin. Both species are associated with warm tropical surface waters and areas with shallow and strong thermoclines. However, ES dolphins are more strongly linked with low-productivity, warm surface waters than NEPS dolphins (Reilly \& Fiedler 1994, Fiedler 2002). The greater affinity of ES dolphins to waters with physical characteristics similar to those present during El Niño conditions may explain why reproductive output in this population is less affected by ENSOgenerated environmental changes.

\section{Length at disassociation}

Length at disassociation, as measured in the present study, can be viewed as a proxy for age at weaning. It is inversely related to birth rate because delphinid calves generally tend to stay closely associated with their mothers until the next pregnancy or birth (Herzing 1997, Mann et al. 2000) or, if the calf dies before it is weaned, until its death. The negative relationship between length at disassociation and number of dolphin sets for NEPS dolphins could be interpreted as a decrease in age at weaning from either increased birth rate or increased calf mortality rates concomitant with an increasing number of dolphin sets. The absence of a temporal trend in length at disassociation for either species means that the decline in proportion with calves over time is not due to changes in calving interval. Thus, the temporal decline in proportion with calves is attributed to increased calf mortality, decreased birth rate, or a combination of the 2 .

The age distribution of NEPS calves measured suggests this population is experiencing depressed reproductive output relative to the ES population. There was a relative dearth of very young NEPS dolphin calves sampled in the length at disassociation analysis: only $17 \%$ of NEPS dolphin calves sampled were $<2$ yr old ( $<126 \mathrm{~cm}$; S. Chivers unpubl. data) (Fig. 6) compared to $85 \%$ of ES dolphins (<144 cm; J. Larese unpubl. data) (Fig. 7). Fewer young calves could be a result of decreased birth rate and/or increased calf mortality. While the small number of very young NEPS dolphins measured could be an artifact of the species' darker body coloration making them harder to detect than ES dolphins, the swimming behavior of very young calves make this explanation unlikely (see 'Potential sampling biases').

For both species, lengths and ages at calf disassociation estimated in the present study exceeded lengths and ages at weaning estimated from fishery data. Archer \& Robertson (2004) estimated length at weaning for data including NEPS, as well as the westernsouthwestern stock of NEPS dolphins (Dizon et al. 1994) by analyzing calves killed in tuna purse-seine nets. This estimate, defined as the length at which there was a 0.5 probability of finding milk in a calf's stomach, was $122 \mathrm{~cm}$ (roughly 2 yr for the NEPS dolphin; S. J. Chivers unpubl. data). Henderson et al. (1980) estimated ages at weaning of 11 mo for ES dolphins and from 1.7 to $2.9 \mathrm{yr}$ for NEPS dolphins, based on the distribution of calf lengths and number of lactating females in the kill. In comparison, the length at disassociation analysis determined that both ES and NEPS dolphin calves were weaned at older ages, at roughly 2 yr and from roughly 3 to $7 \mathrm{yr}$, respectively. The age at weaning estimates from fishery data are probably underestimates, as calves are under-represented in the kill (Archer et al. 2001). The stomach contents analysis is further biased downwards due to the difficulties associated with detecting milk in a calf's stomach (Oftedal 1997, Best 1999). As both wild and captive delphinid calves have not been observed associating with their mother post-weaning (Herzing 1997, Mann et al. 2000), the length at disassociation can be interpreted as length (age) at weaning. In fact, the length at disassociation estimates for NEPS are similar to ages at weaning estimates from those longitudinal 
behavioral studies of wild Atlantic NEPS dolphin calves, which were observed nursing until age 3 to $4 \mathrm{yr}$ on average (Herzing 1997).

\section{Potential sampling biases}

Conditions such as glare from the sun, vertical overlap of dolphins, and water surface disturbances resulted in up to $70 \%$ of the animals photographed in some schools being excluded from analysis. This could possibly lead to sampling biases in the proportion with calves and length at disassociation analyses. Calves older than 2 wk and up to a year swim oriented below the mother in the so-called 'infant position' the majority of the time, at least in bottlenose dolphins (Gubbins et al. 1999, Mann \& Smuts 1999). Calves would probably be less likely to be detected in this position, and could result in a negative bias in the proportion with calves counted and/or in the length (age) distribution of calves measured.

Multiple factors suggest that sampling biases were minimal. Mother-calf pairs probably spend more time near the surface than other dolphins, making them more likely to be counted and measured. In addition, many of the schools photographed were traveling at high speeds to evade the research vessel and/or helicopter. Because dolphins respire more frequently when running than when resting, and swimming in infant position increases the distance traveled to take a breath at the surface, infant-position swimming would be reduced in these schools (Weihs 2004). Although not all of the calves counted could also be measured, a length bias within the sample of calves measured is unlikely.

\section{Implications for the tuna-dolphin issue}

Assessment models (Wade et al. 2007) estimate that NEPS dolphins are at $19 \%$ and ES dolphins at $29 \%$ of their pre-1959 abundance levels, the year that the yellowfin tuna purse-seine fishery began setting on dolphin schools. Given these reduced population sizes and the current low level of reported dolphin bycatch, recovery of both populations would be expected, but has not yet occurred (Gerrodette \& Forcada 2005). Wade et al. (2007) tested hypotheses of fishery effects and habitat changes for the lack of recovery, but came to no clear conclusions based on reported bycatch and estimated dolphin abundance data. Similarly, using aerial photographic data, we modeled the effects of habitat change (as summarized in annual values of the multivariate ENSO index MEI) and fishing effort (as summarized by the annual number of total dolphin sets) on 2 measures of reproductive output, the proportion of adults with calves and calf length at disassociation from its mother. The proportion of NEPS dolphins with calves was affected by MEI. Although interesting in its own right, a response in reproductive output to ENSO conditions does not address the possible role of long-term ecosystem changes in the lack of recovery of this species, as ENSO cycles are a normal feature of the ETP environment.

However, the effect of dolphin sets on both measures of reproduction for NEPS dolphins demonstrates that the practice of setting on dolphins has population-level effects beyond the direct kill recorded by observers on fishing vessels. The decline in proportion with calves and increased length at disassociation with number of dolphin sets could be caused by stress (Myrick \& Perkins 1995, Curry 1999, Reilly et al. 2005), increased predation (Perryman \& Foster 1980), separation of mothers and calves (Archer et al. 2001, Weihs 2004, Edwards 2006), or induced abortion (Perrin et al. 2003, Chivers unpubl. data) resulting from the chase and encirclement procedure. In retrospect, previous studies can be interpreted as showing negative relationships between fishing activity and dolphin reproductive rates. Perrin \& Henderson (1984) for ES dolphins and Barlow (1985) and Chivers \& Myrick (1993) for NEPS dolphins interpreted their results as contrary to density-dependent expectations: the more heavily fished, and therefore more depleted, dolphin populations were expected to have higher reproductive rates and younger ages at sexual maturity, but did not. However, their results, as well as ours, are consistent with the hypothesis that the tuna purse-seine fishery has a negative effect on dolphin reproduction.

Neither measure of reproductive output was related to the number of dolphin sets for ES dolphins. It may be that ES dolphins are less sensitive to the effects of fishing. However, the available measure of fishing activity, total annual number of dolphin sets, primarily reflects trends in fishing activity on NEPS dolphins, since approximately $90 \%$ of dolphin sets involve that species (compared to $44 \%$ of sets for ES dolphins; Archer et al. 2002). Therefore, the reason that the number of dolphin sets was not a predictor for measures of reproduction for ES dolphins may simply be that the measure did not adequately capture the effect of the fishery on that species.

The temporal declines in dolphin reproductive output (proportion with calves) estimated in the present study are the proximate cause, or at least one of the proximate causes, of the lack of recovery of both populations. However, the ultimate cause of these declines is not clear. The temporal decline in proportion with calves over and beyond that explained by the predictor variables means that there are factors affecting repro- 
ductive output other than the ones we modeled. The decline is not likely due to a density-dependent response, as discussed above. The decline may be due to some long-term ecosystem change, although no such change has yet been identified (Fiedler 2002, Reilly et al. 2005). The decline might also be due to some effect of the fishery not captured by the simple measure of annual total dolphin sets. An improved analysis would use a stock-specific number of dolphin sets and have greater spatial and temporal resolution of fishing activity. If reproductive declines are due to the effects of the fishery, it may then be possible to identify specific times and/or areas where effects are greatest and where management actions can be targeted.

Any proposed changes in the management of the yellowfin tuna fishery must consider that fishing methods that do not involve setting on dolphin schools result in bycatch of other depleted marine organisms, including billfish, sea turtles and sharks (Hall 1998, Hinke et al. 2004). Modifications to fishing procedures and gear and/or spatio-temporal restrictions on fishing effort are tools that can be used to prevent the further depletion of dolphin, turtle, and fish populations and to move towards sustainable ecosystem-based management of the yellowfin tuna fishery in particular and ETP fisheries in general.

Acknowledgements. The authors sincerely thank F. I. Archer, S. J. Chivers, W. F. Perrin, E. F. Edwards, J. Larese, E. Eilers, A. Allen, C. Stinchcomb, J. Brandon, M. S. Lynn, J. W. Gilpatrick, N. Kellar, and the scientists and crew aboard the $\mathrm{R} / \mathrm{V}$ 'David Starr Jordan' for their part in the study. Janet Mann and 3 anonymous reviewers provided insightful comments that improved the manuscript.

\section{LITERATURE CITED}

Akaike H (1973) Information theory and an extension of the maximum likelihood principle. In: Petrov BN, Csaki F (eds) 2nd Int Symp on Information Theory. Akademiai Kiado, Budapest, p 267-281

Archer FI, Robertson KM (2004) Age and length at weaning and development of diet of pantropical spotted dolphins, Stenella attenuata, from the eastern tropical Pacific. Mar Mamm Sci 20:232-245

Archer FI, Gerrodette T, Dizon A, Abella K, Southern S (2001) Unobserved kill of nursing dolphin calves in a tuna purseseine fishery. Mar Mamm Sci 17:540-554

Archer FI, Gerrodette T, Jackson A (2002) Preliminary estimates of the annual number of sets, number of dolphins chased, and number of dolphins captured by stock in the tuna purse-seine fishery in the eastern tropical Pacific, 1971-2000. Southwest Fisheries Center Administrative Report LJ-02-10, Southwest Fisheries Science Center, La Jolla, CA. Available at: http://swfsc.noaa.gov/ uploadedFiles/Divisions/PRD/Programs/ETP_Cetacean Assessment/LJ_02_10.pdf (accessed September 2008)

Archer FI, Gerrodette T, Chivers S, Jackson A (2004) Annual estimates of the unobserved incidental kill of pantropical spotted dolphin (Stenella attenuata attenuata) calves in the tuna purse-seine fishery of the eastern tropical Pacific. Fish Bull (Wash DC) 102:233-244

Barlow J (1985) Variability, biases, and trends in reproductive rates of spotted dolphins, Stenella attenuata. Fish Bull (Wash DC) 83:657-669

Barlow J, Hohn A (1984) Interpreting spotted dolphin age distributions. Southwest Fisheries Center Technical Memorandum NOAA-TM-NMFS-SWFC-48, Southwest Fisheries Science Center, La Jolla, CA

Bayliff WH (ed) (2004) 2002 annual report. Inter-American Tropical Tuna Commission, La Jolla, CA

Best PB (1999) Food and feeding of sperm whales Physeter macrocephalus off the west coast of South Africa. S Afr J Mar Sci 21:393-413

Bright AM, Chivers SJ (1991) Postnatal growth rates: a comparison of northern and southern stocks of offshore spotted dolphin, Stenella attenuata. NOAA-NMFS-SWFSC Administrative Report LJ-91-30, Southwest Fisheries Science Center, La Jolla, CA

Burnham KP, Anderson DR (1998) Model selection and inference: a practical information-theoretic approach. Springer-Verlag, New York

Chivers SJ, Myrick AC (1993) Comparison of age at sexual maturity and other reproductive parameters for two stocks of spotted dolphin, Stenella attenuata. Fish Bull (Wash DC) 91:611-618

Chivers SJ, Scott MD (2002) Tagging and tracking of Stenella spp. during the 2001 Chase Encirclement Stress Studies Cruise. SWFSC Administrative Report LJ-02-33, Southwest Fisheries Science Center, La Jolla, CA. Available at: http://swfsc.noaa.gov/uploadedFiles/Divisions/ PRD/Programs/ETP_Cetacean_Assessment/LJ_02_33.pdf (accessed September 2008)

Curry BE (1999) Stress in mammals: the potential influence of fishery-induced stress on dolphins in the eastern tropical Pacific Ocean. NOAA Tech Memo NMFS-SWFSC-260, NOAA, La Jolla. Available at: http://swfsc.noaa.gov/publications/TM/SWFSC/NOAA-TM-NMFS-SWFSC-260.pdf (accessed September 2008)

Dizon AE, Perrin WF, Akin PA (1994) Stocks of dolphins (Stenella spp. and Delphinus delphis) in the eastern tropical Pacific: a phylogeographic classification. NOAA Tech Rep, NMFS 119, US Department of Commerce, Seattle, WA

Edwards EF (2006) Duration of unassisted swimming activity for spotted dolphin (Stenella attenuata) calves: implications for mother-calf separation during tuna purse-seine sets. Fish Bull (Wash DC) 104:125-135

Fiedler PC (2002) Environmental change in the eastern tropical Pacific Ocean: review of ENSO and decadal variability. Mar Ecol Prog Ser 244:265-283

Gerrodette T (2002) Tuna-dolphin issue. In: Perrin WF, Wursig B, Thewissen JGM (eds) Encyclopedia of marine mammals. Academic Press, San Diego, CA, p 1269-1273

> Gerrodette T, Forcada J (2005) Non-recovery of two spotted and spinner dolphin populations in the eastern tropical Pacific Ocean. Mar Ecol Prog Ser 291:1-21

Gerrodette T, Perryman WL, Barlow J (2002) Calibrating group size estimates of dolphins in the eastern tropical Pacific Ocean. SWFSC Admininstrative Report LJ-02-08, Southwest Fisheries Science Center, La Jolla, CA. Available at: http://swfsc.noaa.gov/uploadedFiles/Divisions/ PRD/Programs/ETP_Cetacean_Assessment/LJ_02_08.pdf (accessed September 2008)

Gilpatrick JW (1996) Calibration of radar altimeter readings 
used in aerial photogrammetry of eastern tropical Pacific dolphins 1992 and 1993. NOAA Technical Memorandum, NOAA-TM-NMFS-SWFSC-226, Available at: http://swfsc. noaa.gov/publications/TM/SWFSC/NOAA-TM-NMFSSWFSC-226.pdf (accessed September 2008)

Goodman D (1984) Statistics for reproductive rate estimates, and their implications for population projection. Reports of the International Whaling Commission 6(Spec Issue): 161-173

Gosliner ML (1999) The tuna-dolphin controversy. In: Twiss JR Jr, Reeves RR (eds) Conservation and management of marine mammals. Smithsonian Institution Press, Washington, DC, p 120-1555

> Gubbins C, McCowan B, Lynn SK, Hooper S, Reiss D (1999) Mother-infant spatial relations in captive bottlenose dolphins, Tursiops truncatus. Mar Mamm Sci 15:751-765

Hall MA (1998) An ecological view of the tuna-dolphin problem: impacts and trade-offs. Rev Fish Biol Fish 8(1):1-34

Hastie T, Tibshirani R (1990) Generalized additive models. Chapman \& Hall, London

- Hedley C (2001) The 1998 agreement on the International Dolphin Conservation Program: recent developments in the tuna-dolphin controversy in the eastern Pacific Ocean. Ocean Dev Int Law 32:71-92

Henderson JR, Perrin WF, Miller RB (1980) Rate of gross annual production in dolphin populations (Stenella spp. and Delphinus delphis) in the eastern tropical Pacific, 1973-1978. SWFSC Administrative Report LJ-80-02, Southwest Fisheries Science Center, La Jolla, CA,

Herzing DL (1997) The life history of free-ranging Atlantic spotted dolphins (Stenella frontalis): age classes, color phases, and female reproduction. Mar Mamm Sci 13: 576-595

Hinke JT, Kaplan IC, Aydin K, Watters GM, Olson RJ, Kitchell JF (2004) Visualizing the food-web effects of fishing for tunas in the Pacific Ocean. Ecol Soc 9(1):10. Available at: www.ecologyandsociety.org/vol9/iss1/art10

Hoyle SD, Maunder MN (2004) A Bayesian integrated population dynamics model to analyze data for protected species. Anim Biodivers Conserv 27:247-266

Joseph J (1994) The tuna-dolphin controversy in the eastern Pacific Ocean: biological, economic, and political impacts. Ocean Dev Int Law 25:1-30

Mann J, Smuts BB (1999) Behavioral development in wild bottlenose dolphin newborns (Tursiops sp.). Behaviour 136: 529-566

Mann J, Conner RC, Barre LM, Heithaus MR (2000) Female reproductive success in bottlenose dolphins (Tursiops $\mathrm{sp}$.): life history, habitat, provisioning, and group-size effects. Behav Ecol 11:210-219

Moran PAP (1950) Notes on continuous stochastic phenomena. Biometrika 37:17-23

Myrick AC, Perkins PC (1995) Adrenocortical color darkness and correlates as indicators of continuous acute premortem stress in chased and purse-seine captured male dolphins. Pathophysiology 2:191-204

Myrick AC, Hohn AA, Barlow J, Sloan PA (1986) Reproductive biology of female spotted dolphins, Stenella attenuata, from the eastern tropical Pacific. Fish Bull (Wash DC) 84:247-259

Noren SR, Edwards EF (2007) Physiological and behavioral development of delphinid calves: implications for calf separation and morality due do tune purse-seine sets. Mar Mamm Sci 23:15-29

Norris KS, Dohl TP (1980) Behavior of the Hawaiian spinner dolphin, Stenella longirostris. Fish Bull (Wash DC) 77: 821-849
Norris KS, Johnson CM (1994) Schools and schooling. In: Norris KS, Wursig B, Wells RS, Wursig M, Brownlee SM, Johnson CM, Solow J (eds) The Hawaiian spinner dolphin. University of California Press, Berkeley, CA, p 232-242

- Oftedal OT (1997) Lactation in whales and dolphins: evidence of divergence between baleen- and toothed-species. J Mammary Gland Biol Neoplasia 2:205-230

Perrin WF (1969) Using porpoise to catch tuna. World Fish 18: $42-45$

Perrin WF, Henderson JR (1984) Growth and reproductive rates in two populations of spinner dolphins, Stenella longirostris, with different histories of exploitation. Reports of the International Whaling Commission 6(Spec Issue): 417-430

Perrin WF, Coe JM, Zweifel JR (1976) Growth and reproduction of the spotted porpoise, Stenella attenuata, in the eastern tropical Pacific. Fish Bull (Wash DC) 74:229-269

Perrin WF, Holts DB, Miller RB (1977) Growth and reproduction of the eastern spinner dolphin, a geographical form of Stenella longirostris in the eastern tropical Pacific. Fish Bull (Wash DC) 75:725-750

Perrin WF, Chivers SJ, Archer FI II (2003) Fetal mortality in dolphins exploited by the tuna fishery. In: Conference abstracts. Society for Marine Mammology, Greensboro, NC (abstract)

Perryman WL, Foster TC (1980) Preliminary report on predation by small whales, mainly the false killer whale, Pseudorca crassidens, on dolphins (Stenella spp. and Delphinus delphus) in the eastern tropical Pacific. SWFSC Administrative Report LJ-80-05, Southwest Fisheries Science Center, La Jolla, CA

> Perryman WL, Lynn MS (1993) Identification of geographic forms of common dolphin (Delphinus delphis) from aerial photogrammetry. Mar Mamm Sci 9:119-137

Perryman WL, Lynn MS (1994) Examination of stock and school structure of striped dolphins (Stenella coeruleoalba) in the eastern Pacific from aerial photogrammetry. Fish Bull (Wash DC) 92:122-131

> Perryman WL, Westlake RL (1998) A new geographic form of the spinner dolphin, Stenella longirostris, detected with aerial photogrammetry. Mar Mamm Sci 14:38?50

Powers JE, Barlow J (1979) Biases in the tuna-net sampling of dolphins in the eastern tropical Pacific. Preliminary report for the Status of Porpoise Stocks workshop, August 27-31

Reilly SB, Fiedler PC (1994) Interannual variability of dolphin habitats in the eastern tropical Pacific. I. Research vessel surveys, 1986-1990. Fish Bull (Wash DC) 92: $434-450$

Reilly SB, Donahue MA, Gerrodette T, Forney K and others (2005) Report of the scientific research program under the International Dolphin Conservation Program Act. NOAA Tech Memo NMFS-SWFSC-372, NOAA, La Jolla, CA. Available at: http://reefshark.nmfs.noaa.gov/pr/saip/pdfs/ techmemo372.pdf (accessed September 2008)

Schnute JT, Hilborn R (1993) Analysis of contradictory data sources in fish stock assessment. Can J Fish Aquat Sci 50: $1916-1923$

Scott MD (1998) Diel patterns in aggregations of pelagic dolphins and tunas in the eastern Pacific. Mar Mamm Sci 14: $401-428$

Smith TD (1983) Changes in size of three dolphin (Stenella spp.) populations in the eastern tropical Pacific. Fish Bull (Wash DC) 81:1-13

Wade PR (1993) Estimation of historical population size of the eastern spinner dolphin (Stenella longirostris orientalis). Fish Bull (Wash DC) 91:775-787

Wade PR (1995) Revised estimates of incidental kill of dol- 
phins (Delphinidae) by the purse-seine tuna fishery in the eastern tropical Pacific, 1959-1972. Fish Bull (Wash DC) 93:345-354

> Wade PR, Watters GM, Gerrodette T, Reilly SB (2007) Depletion of spotted and spinner dolphins in the eastern tropical Pacific: modeling hypotheses for their lack of recovery. Mar Ecol Prog Ser 343:1-14

Ward EJ (2005) Differences between fishery-dependent and fishery-independent estimates of single- and mixedspecies dolphin schools: implications for single-species stock assessments. Mar Mamm Sci 21:189-203

Editorial responsibility: Rory Wilson,

Swansea, UK
Weihs D (2004) The hydrodynamics of dolphin drafting. J Biol 3:8

Wolter K, Timlin MS (1993) Monitoring ENSO in COADS with a seasonally adjusted principal component index. In: Proc 17th Clim Diagn workshop. NOAA/N MC/CAC, NSSL, Oklahoma Climate Survey, CIMMS and the School of Meteorology, University of Oklahoma, Norman, OK, p 52-57. Available at: www.cdc.noaa.gov/people/klaus. wolter/MEI/WT1.pdf (accessed September 2008)

Wolter K, Timlin MS (1998) Measuring the strength of ENSO events-How does 1997/98 rank? Weather 53:315-324

Submitted: November 19, 2007; Accepted: June 11, 2008 Proofs received from author(s): September 25, 2008 